BRAVZULIAN JOURNAL

OF MEDICAL AND BIOLOGICAL RESH.ARCH

www.bjournal.com.br
ISSN 0100-879X

Volume 44 (7) 606-728 July 2011

BIOMEDICAL SCIENCES

AND

CLINICAL INVESTIGATION

Braz J Med Biol Res, July 2011, Volume 44(7) 707-712

doi: 10.1590/S0100-879X2011007500069

Immunological and biochemical parameters of patients with metabolic syndrome and the participation of oxidative and nitroactive stress

A.N.C. Simão, M.A.B. Lozovoy, T.N.C. Simão, D. Venturini, D.S. Barbosa, J.B. Dichi, T. Matsuo, R. Cecchini and I. Dichi

The Brazilian Journal of Medical and Biological Research is partially financed by

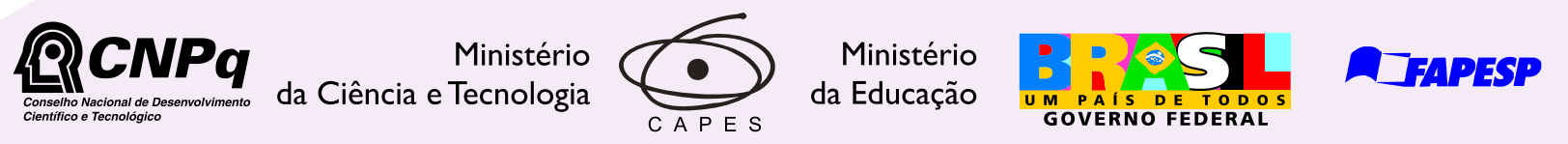

Institutional Sponsors
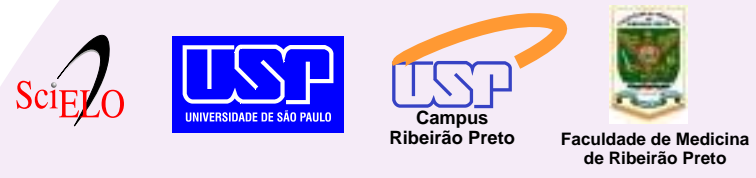
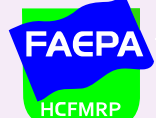

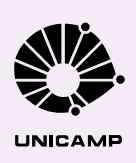

$\oplus$ SHIMADZU

GE Healthcare
Hotsite of proteomics metabolomics developped by:

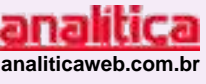

Thermo SCIENTIFIC 


\title{
Immunological and biochemical parameters of patients with metabolic syndrome and the participation of oxidative and nitroactive stress
}

\author{
A.N.C. Simão ${ }^{1}$, M.A.B. Lozovoy ${ }^{2}$, T.N.C. Simão ${ }^{3}$, D. Venturini ${ }^{1}$, D.S. Barbosa ${ }^{1}$, \\ J.B. Dichi ${ }^{4}$, T. Matsuo ${ }^{5}$, R. Cecchini ${ }^{6}$ and I. Dichi ${ }^{4}$ \\ ${ }^{1}$ Departamento de Patologia, Análises Clínicas e Toxicológicas, \\ Universidade Estadual de Londrina, Londrina, PR, Brasil \\ ${ }^{2}$ Departamento de Análises Clínicas, ${ }^{3}$ Departamento de Nutrição Clínica, \\ Universidade Norte do Paraná, Londrina, PR, Brasil \\ ${ }^{4}$ Departamento de Clínica Médica, ${ }^{5}$ Departamento de Estatística, \\ ${ }^{6}$ Departamento de Ciências Patológicas, Universidade Estadual de Londrina, Londrina, PR, Brasil
}

\begin{abstract}
Metabolic syndrome (MS) is a multifactorial disease involving inflammatory activity and endothelial dysfunction. The aim of the present study was to evaluate the relationship between the changes in lipoperoxidation, in immunological and biochemical parameters and in nitric oxide metabolite (NOx) levels in MS patients. Fifty patients with MS (4 males/46 females) and 50 controls (3 males/47 females) were studied. Compared to control (Mann-Whitney test), MS patients presented higher serum levels ( $\mathrm{P}<0.05)$ of fibrinogen: $314(185-489)$ vs 262 (188-314) mg/dL, C-reactive protein (CRP): 7.80 (1.10-46.50) vs 0.70 (0.16-5.20) mg/dL, interleukin-6: 3.96 (3.04-28.18) vs $3.33(2.55-9.63) \mathrm{pg} / \mathrm{mL}$, uric acid: 5.45 (3.15-9.65) vs 3.81 (2.70-5.90) $\mathrm{mg} / \mathrm{dL}$, and hydroperoxides: $20,689(19,076-67,182)$ vs 18,636 $(15,926-19,731) \mathrm{cpm}$. In contrast, they presented lower $(\mathrm{P}<$ $0.05)$ adiponectin: 7.11 (3.19-18.22) vs 12.31 (9.11-27.27) $\mathrm{gg} / \mathrm{mL}$, and NOx levels: 5.69 (2.36-8.18) vs 6.72 (5.14-12.43) $\mu \mathrm{M}$. NOx was inversely associated (Spearman's rank correlation) with body mass index $(r=-0.2858, P=0.0191)$, insulin resistance determined by the homeostasis model assessment $(r=-0.2530, P=0.0315)$, CRP $(r=-0.2843, P=0.0171)$ and fibrinogen $(r$ $=-0.2464, P=0.0413)$, and positively correlated with hydroperoxides $(r=0.2506, P=0.0408)$. In conclusion, NOx levels are associated with obesity, insulin resistance, oxidative stress, and inflammatory markers. The high uric acid levels together with reactive oxygen species generation may be responsible for the reduced NO levels, which in turn lead to endothelial dysfunction. The elevated plasma chemiluminescence reflecting both increased plasma oxidation and reduced antioxidant capacity may play a role in the MS mechanism.
\end{abstract}

Key words: Metabolic syndrome; Uric acid; Inflammation; Adiponectin; Nitric oxide; Lipoperoxidation

\section{Introduction}

Metabolic syndrome (MS) consists of pathological conditions that include insulin resistance, arterial hypertension, obesity, and dyslipidemia (1); associated abnormalities include inflammation and endothelial dysfunction (2).

Vascular dysfunction and damage have been shown to be associated with impaired endothelial nitric oxide (NO) metabolism and function. Endothelial dysfunction is considered to be a hallmark in the pathophysiology of $\mathrm{MS}$, and can be evaluated by several means, including the assessment of NO metabolite (NOx) levels (3). NO is synthesized in endothelial cells by endothelial nitric oxide synthase (eNOS) activity, and is responsible for vasodilatation and for the maintenance of endothelial function; eNOS is expressed constitutively and synthesizes NO in only small amounts under basal conditions. NO production may also be stimulated by an increase in inducible nitric oxide synthase (iNOS) expression provoked, for instance, by oxidative stress or pro-inflammatory cytokines (4). Therefore, iNOSderived NO mediates the inflammatory response and has been shown to cause vascular dysfunction in a number of experimental models (5).

A myriad of pathological factors, such as insulin resis-

Correspondence: A.N.C. Simão, Departamento de Patologia, Análises Clínicas e Toxicológicas, Universidade Estadual de Londrina, Avenida Robert Koch, 60, 86038-440 Londrina, PR, Brasil. E-mail: deianame@yahoo.com.br

Received May 17, 2010. Accepted May 13, 2011. Available online May 27, 2011. Published July 25, 2011. 
tance, hyperuricemia, inflammation, hypoadiponectinemia, and oxidative stress could lead to a decrease in NO bioavailability $(3,6)$.

Although some studies have shown association between components of metabolic syndrome and NO, the data are scarce and contradictory. Furthermore, to our knowledge, the interrelationship between serum NO and insulin resistance, uric acid values, adiponectinemia, pro-inflammatory markers, oxidative stress, and obesity has not been evaluated simultaneously thus far. These relationships could contribute to the understanding of the pathophysiology of endothelial dysfunction in MS. Thus, the aim of the present study was to evaluate a possible relationship between the changes in lipoperoxidation, immunological, and biochemical parameters observed in MS patients and NOx levels.

\section{Material and Methods}

\section{Subjects \\ One hundred individuals selected among Internal Medicine ambulatory patients and healthy workers of the Hospital Uni- versitário de Londrina, PR, Brazil, were chosen to participate in this transverse study. Control and MS subjects were paired for gender, age, race, smoking, and alcohol intake; the control group included 50 healthy individuals with body mass indexes (BMI) between 20 and $25 \mathrm{~kg} / \mathrm{m}^{2}$ and without MS. Information about lifestyle factors and medical history was obtained by clinical evaluation. MS was defined according to Adult Treat- ment Panel III criteria. MS was diagnosed when three of five of the following characteristics were detected: 1) abdominal obesity, waist circumference $\geq 102 \mathrm{~cm}$ in men and $\geq 88 \mathrm{~cm}$ in women; 2) hypertriglyceridemia $\geq 150 \mathrm{mg} / \mathrm{dL}$ (1.695 mM); 3) low levels of high-density lipoprotein (HDL) cholesterol: $\leq 40 \mathrm{mg} / \mathrm{dL}$ (1.036 mM) in men and $\leq 50 \mathrm{mg} / \mathrm{dL}(1.295 \mathrm{mM})$ in women; 4) high blood pressure: $\geq 130 / 85 \mathrm{mmHg} ; 5$ ) high fasting glucose: $\geq 110 \mathrm{mg} / \mathrm{dL}$ (7). None of the participants in the study presented thyroid, renal, hepatic, gastrointestinal, or oncological disease, and none had a clinically evident infection or were receiving drugs for hyperglycemia or drugs known to affect lipoprotein and uric acid metabolism or inflammatory markers, for at least 4 weeks before the study. All patients gave written informed consent to participate, and the study protocol was approved (CEP 298/05) by the Ethics Committee of Universidade de Londrina (PR, Brazil).}

\section{Anthropometric and blood pressure measurements \\ Height and weight were measured in the morning with subjects wearing light clothing, but no shoes. After 5 min of rest, each subject had his/her blood pressure measured on the left arm while in a sitting position. We considered the current use of antihypertensive medication as an indication of high blood pressure. BMI was calculated as weight $(\mathrm{kg})$ divided by height $(\mathrm{m})$ squared. Waist circumference was measured with a soft tape on standing subjects midway}

between the lowest rib and the iliac crest.

\section{Determination of biochemical biomarkers}

After fasting for $12 \mathrm{~h}$, the patients underwent the following laboratory blood analysis: glucose, total cholesterol, HDL cholesterol, low-density lipoprotein cholesterol (LDL), triacylglycerol (TG), and uric acid were evaluated with a biochemical auto-analyzer (Dimension Dade AR Dade Behring, USA) using Dade Behring ${ }^{\circledR}$ kits. Plasma insulin levels were determined by MEIA(AXSYM, Abbott ${ }^{\circledR}$ Laboratory, Germany). All samples were centrifuged at $1950 \mathrm{~g}$ for $15 \mathrm{~min}$, and plasma or serum aliquots were stored at $-70^{\circ} \mathrm{C}$ until assayed. Interassay and intra-assay CVs were $<10 \%$ for all assays as determined with human serum.

The homeostasis model assessment of insulin resistance (HOMA-IR) was used as a surrogate measure of insulin sensitivity (8). HOMA-IR = insulin fasting $(\mu \mathrm{U} / \mathrm{mL})$ $x$ glucose fasting $(\mathrm{mM}) / 22.5$.

\section{Determination of inflammatory and immunological parameters}

Fibrinogen was measured by the Klauss method, and serum highly sensitive $\mathrm{C}$-reactive protein (CRP) was measured using a nephelometric assay (Behring Nephelometer II, Dade Behring); serum tumor necrosis factor- $\alpha$ (TNF- $\alpha$ ), interleukin-6 (IL-6), and adiponectin were measured by a sandwich enzyme-linked immunosorbent assay (ELISA) using a commercial immunoassay (R\&D Systems, USA). Interassay and intra-assay CVs were $<10 \%$ for all assays as determined with human serum.

\section{NOx levels}

Serum NO levels were assessed on the basis of nitrite $\left(\mathrm{NO}_{2}{ }^{-}\right)$and nitrate $\left(\mathrm{NO}_{3}{ }^{-}\right)$concentration according to the Griess reaction supplemented by the reduction of nitrate to nitrite with cadmium $(9,10)$. The results are reported as $\mu \mathrm{M}$.

\section{Analysis of tert-butyl-hydroperoxide-initiated chemiluminescence}

Plasma tert-butyl-hydroperoxide-initiated chemiluminescence (CL-LOOH) was determined as described by Gonzalez et al. (11). CL is considered to be much more sensitive and specific (12) than the thiobarbituric acid reactive substances (TBARS) method (13), the method usually employed to determine hydroperoxides. The results are reported as counts per minute.

\section{Statistical analysis}

Data are reported as the median, minimum and maximum. Data for control subjects and patients with MS were compared using the Mann-Whitney test. Correlations were determined by Spearman's rank correlation. The results were considered to be significant when $P<0.05$. A statistical analysis program (Graph Pad InStat, Graph Pad Software, Inc.) was used. 


\section{Results}

The clinical and biochemical characteristics of the study subjects are shown in Table 1. There were no differences between groups regarding age, gender, or smoking habit. The group of MS patients had significantly higher BMI, waist circumference, systolic and diastolic blood pressure, TG levels, fasting glucose, insulin, HOMA, and uric acid levels compared to controls $(P<0.0001)$. The MS group also had significantly lower HDL-cholesterol levels $(\mathrm{P}<$ $0.0001)$ and serum NOx levels $(P=0.0480)$ compared to controls (Table 1).

Regarding the levels of the inflammatory markers in MS patients compared to controls, $\operatorname{CRP}(P<0.0001)$, fibrinogen $(P=$ $0.0087)$, and serum IL-6 ( $P=0.0078)$ concentrations were significantly higher. On the other hand, adiponectin levels were significantly lower in the MS group ( $P=0.0261)$. TNF- $\alpha$ levels were not statistically different between groups. Evaluated by CL, lipid hydroperoxide levels were significantly higher in the MS patients $(P<0.0001$; Table 2$)$.

Serum NOx levels were inversely correlated with $\mathrm{BMI}(r=-0.2858, \mathrm{P}=0.0191$; Figure $1 \mathrm{~A}$ ) and showed the same relationship with waist circumference $(r=-0.2207$, $P=0.0684$ ) (data not shown). NO concentration did not correlate with serum fasting insulin levels (data not shown), but it was inversely associated with insulin resistance evaluated by HOMA-IR ( $r=-0.2573, P=$ 0.0315 ; Figure 1B).

NOx was inversely correlated with fibrinogen $(r=-0.2464, P=0.0413)$ and $C R P$ $(r=-0.2843, P=0.0171)$; however, it was not associated with adiponectin or with uric acid levels (Table 3). On the other hand, NOx was positively correlated with oxidative stress measured by lipid hydroperoxide levels $(r=0.2506, P=0.0408$; Figure $1 \mathrm{C}$ ). Hydroperoxide levels were not associated with any MS component (data not shown).

\section{Discussion}

The present study showed that serum NOx levels in patients with MS are associated with insulin resistance, obesity, inflammatory status, and oxidative stress, but not with serum uric acid or adiponectin levels. As expected, all parameters known to be related to MS were significantly different in the group of patients compared to controls. There was also a decrease in serum NOx ney test). levels and an increase in oxidative stress evaluated by chemiluminescence in the group of patients.

Our data agree with other studies $(14,15)$ that showed a significant inverse correlation between NOx and BMI and between NOx and insulin resistance measured by HOMA-IR. The same inverse correlation was detected regarding abdominal circumference, showing that obesity and decreases in insulin action are associated with the reduction of serum $\mathrm{NO}$ levels in MS. These associations were not detected in the control group (data not shown). On the other hand, Ghasemi et al. (16) showed a positive relationship between $\mathrm{BMI}$ and NOx values in overweight

Table 1. Comparison of clinical and laboratory characteristics of the patients with metabolic syndrome and controls of the present study.

\begin{tabular}{lcc}
\hline & Controls $(\mathrm{N}=50)$ & Metabolic syndrome $(\mathrm{N}=50)$ \\
\hline Gender (male/female) & $3 / 47$ & $4 / 46$ \\
Age (years) & $42.0(8.4)$ & $45.9(9.8)$ \\
Smoking/No smoking & $5 / 45$ & $6 / 44$ \\
BMI $\left(\mathrm{kg} / \mathrm{m}^{2}\right)$ & $22.9(18.7-24.9)$ & $37.2(27.1-53.9)^{*}$ \\
SBP $(\mathrm{mmHg})$ & $101.0(81.0-130.0)$ & $134.5(89.0-203.0)^{*}$ \\
DBP $(\mathrm{mmHg})$ & $65.0(47.0-80.0)$ & $83.0(53.0-124.0)^{*}$ \\
Waist circumference $(\mathrm{cm})$ & $80.0(61.0-97.0)$ & $110.0(82.0-157.0)^{*}$ \\
Triacylglycerol $(\mathrm{mg} / \mathrm{dL})$ & $87.0(32.0-145.0)$ & $191.0(40.0-491.0)^{*}$ \\
HDL-C $(\mathrm{mg} / \mathrm{dL})$ & $53.0(40.0-77.0)$ & $39.0(19.0-81.0)^{*}$ \\
Fasting insulin $(\mu \mathrm{U} / \mathrm{mL})$ & $6.3(2.9-14.9)$ & $15.6(4.1-75.4)^{*}$ \\
Fasting glucose $(\mathrm{mg} / \mathrm{dL})$ & $78.0(71.0-87.0)$ & $100.0(74.0-247.0)^{*}$ \\
HOMA-IR & $1.22(0.57-4.88)$ & $3.67(0.83-32.24)^{*}$ \\
Uric acid $(\mathrm{mg} / \mathrm{dL})$ & $3.81(2.70-5.90)$ & $5.45(3.15-9.65)^{*}$ \\
NO $(\mu \mathrm{M})$ & $6.72(5.14-12.43)$ & $5.69(2.36-8.18)^{*}$ \\
\hline
\end{tabular}

Data are reported as median (range) except for age, which is reported as mean (SD). $\mathrm{BMI}=$ body mass index; $\mathrm{SBP}=$ systolic blood pressure; $\mathrm{DBP}=$ diastolic blood pressure; HDL-C = high-density lipoprotein cholesterol; HOMA-IR = homeostasis model of assessment of insulin resistance; $\mathrm{NO}=$ nitric oxide. ${ }^{*} \mathrm{P}<0.05$ compared to control (Mann-Whitney test).

Table 2. Inflammatory markers and lipid hydroperoxide levels of the patients with metabolic syndrome and controls.

\begin{tabular}{lcc}
\hline & Controls $(\mathrm{N}=50)$ & Metabolic syndrome $(\mathrm{N}=50)$ \\
\hline Fibrinogen $(\mathrm{mg} / \mathrm{dL})$ & $262.00(188.0-314.0)$ & $314.00(185-489.0)^{*}$ \\
CRP $(\mathrm{mg} / \mathrm{dL})$ & $0.70(0.16-5.20)$ & $7.80(1.10-46.50)^{*}$ \\
TNF- $\alpha(\mathrm{pg} / \mathrm{mL})$ & $41.20(8.57-86.26)$ & $32.68(11.62-193.81)$ \\
$\mathrm{IL}-6(\mathrm{pg} / \mathrm{mL})$ & $3.33(2.55-9.63)$ & $3.96(3.04-28.18)^{*}$ \\
Adiponectin $(\mu \mathrm{g} / \mathrm{mL})$ & $12.31(9.11-27.27)$ & $7.11(3.19-18.22)^{*}$ \\
Hydroperoxides $(\mathrm{cpm})$ & $18,636(15,926-19,731)$ & $20,689(19,076-67,182)^{*}$ \\
\hline
\end{tabular}

Data are reported as median (range). CRP $=$ C-reactive protein; TNF- $\alpha=$ tumor necrosis factor- $\alpha$; IL-6 = interleukin-6. ${ }^{*} \mathrm{P}<0.05$ compared to control (Mann-Whit- 

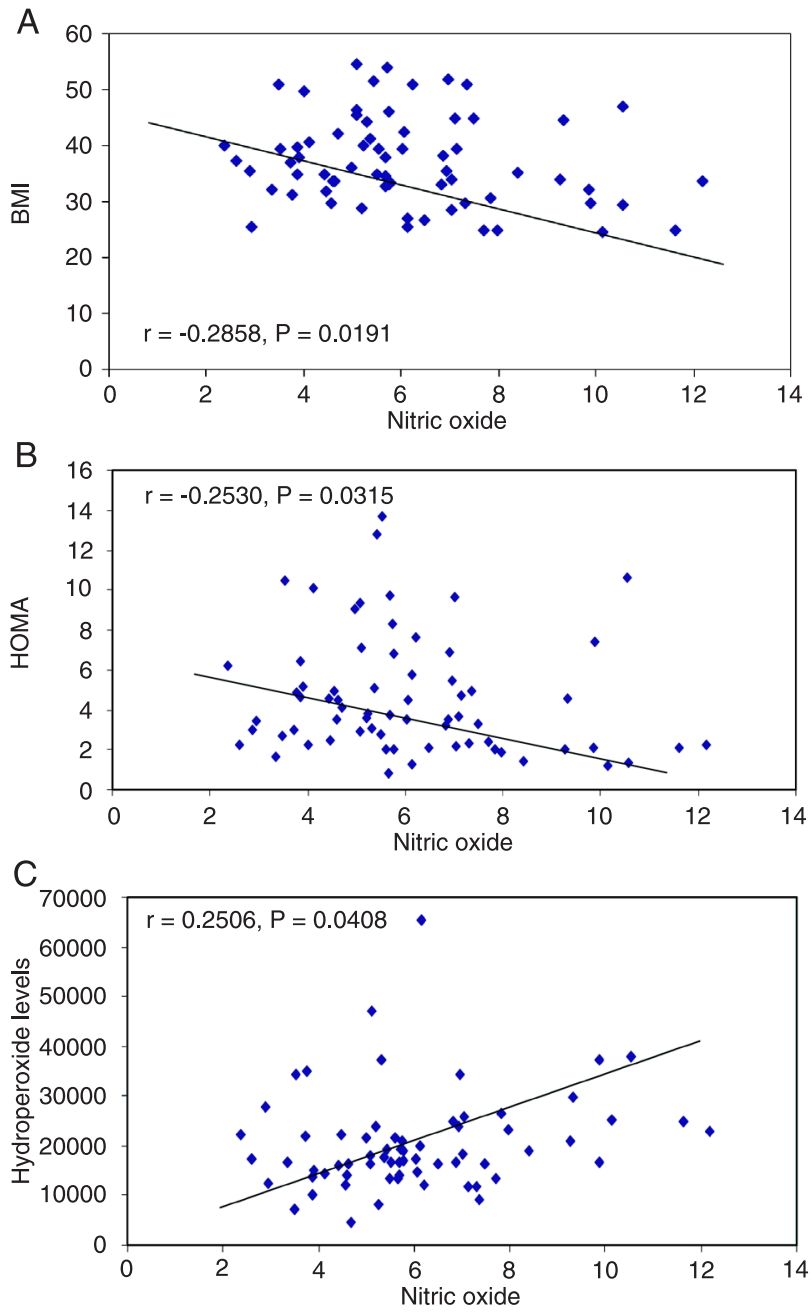

Figure 1. Dispersion graphs between serum nitric oxide levels and $A$, body mass index (BMI), $B$, homeostasis model of assessment (HOMA) and $C$, serum lipid hydroperoxide levels in patients with metabolic syndrome. Data are reported as Spearman's correlation coefficients and $\mathrm{P}$ values.

and obese women, but their patients were less obese than the patients of the present study.

Although endothelial dysfunction has been considered an important issue in patients with MS, results of studies on serum NOx levels in patients with MS have been contradictory $(6,14,15)$. Sun et al. (14) also showed that NOx levels were reduced in MS. However, Zahedi Asl et al. (6) showed higher NOx concentration in subjects with MS and type 2 diabetes. Lin et al. (15) observed that patients with severe obesity and non-obese subjects had similar serum NOx levels.

Oxidative stress mediated by reactive oxygen species (ROS) and reactive nitrogen species has been detected in patients with MS (15,17-19) and in obese patients, and ROS were correlated with serum NOx levels (15). In the present study, MS subjects also showed a significant increase
Table 3. Spearman's analysis of the correlation of plasma nitric oxide (NO) with plasma uric acid and inflammatory markers in patients with metabolic syndrome.

\begin{tabular}{lrc}
\hline & \multicolumn{2}{c}{ NO } \\
\cline { 2 - 3 } & $r$ & $P$ \\
\hline Fibrinogen & -0.246 & 0.041 \\
CRP & -0.284 & 0.017 \\
TNF- $\alpha$ & 0.044 & 0.726 \\
IL-6 & -0.083 & 0.509 \\
Adiponectin & 0.014 & 0.913 \\
Uric acid & -0.018 & 0.889 \\
\hline
\end{tabular}

CRP $=$ C-reactive protein; TNF- $\alpha=$ tumor necrosis factor- $\alpha$; IL-6 = interleukin-6.

in lipid hydroperoxides measured by chemiluminescence that correlated positively with NOx levels. However, this association was not found in the control group (data not shown). Despite this positive association, NOx levels were decreased compared to control. The reduction in NOx levels is coherent with both endothelial dysfunction (20) and the increased oxidative stress found in this study. This statement is supported by the fact that NO is consumed in a reaction with superoxide anion yielding a strong oxidant species, $\mathrm{ONOO}^{-}$, which in turn accelerates the lipid peroxidation reaction $(15,21,22)$. Peroxynitrite production is also supported by the elevated levels of nitrotyrosine, a marker of endogenous peroxynitrite generation found in both human and animal models $(23,24)$. An increased concentration of uric acid may also contribute to the reduced NO, increased oxidative stress and impaired endothelial function found in the present study $(20,25)$, leading to increased risk factors for the development of obesity, hypertension, and cardiovascular disease, which have been associated with oxidative stress (26).

Despite its role as a major antioxidant in plasma, uric acid can form free radicals in various reactions, including peroxynitrite, whose products are responsible for the amplification of lipid oxidation (26). In addition, uric acid has been shown to reduce $\mathrm{NO}$ bioavailability in various cell types via mechanisms involving redox control (27) and also activating arginase and depleting NO (28). Recently, our group observed that serum uric acid levels correlated with several parameters of MS (29). The involvement of uric acid in oxidative and nitroactive stress is very complex because of its antioxidant/prooxidant capacity. Probably this balance is concentration and $\mathrm{pH}$ dependent.

MS is characterized by a low-grade inflammatory state. Although in the current study serum IL-6 levels were significantly higher in MS patients compared to controls, the same did not occur with TNF-a. Adipose tissue, and the visceral depot in particular, is a major contributor to plasma 
IL-6 levels and explains approximately $33 \%$ of the plasma IL- 6 concentration. TNF- $\alpha$ does not seem to be released into the circulation and thus is unable to signal systemically $(30,31)$, functioning as a paracrine pathway (32). In agreement with a previous study (33), our data also showed that serum TNF- $\alpha$ level is not an appropriate inflammatory marker in patients with MS.

Acute-phase reactant proteins, CRP and fibrinogen were increased in patients with MS, and they were inversely related to serum NOx levels. However, this association was not found in the control group (data not shown).

It is conceivable that increased NO consumption occurs with higher inflammatory activity only in morbidly obese patients. In the meantime, acute-phase reactant proteins were also related to waist circumference (data not shown). It could be hypothesized that increased abdominal fat and acute-phase reactant proteins lead to a condition that favors oxidative stress, resulting in an increased consumption of serum NO metabolites. On the other hand, adiponectin levels were significantly decreased in patients with MS. These results agree with the literature, showing that MS patients present a higher pro-inflammatory state and a decrease in anti-inflammatory mediators $(8,33)$.

In the current study, the correlation between adiponectin and serum NO concentration was not observed. Our data agree with those reported by Lin et al. (15), which showed that serum NOx levels were not correlated with adiponectin

\section{References}

1. Reaven GM. Banting lecture 1988. Role of insulin resistance in human disease. Diabetes 1988; 37: 1595-1607.

2. Santos AC, Lopes C, Guimaraes JT, Barros H. Central obesity as a major determinant of increased high-sensitivity C-reactive protein in metabolic syndrome. Int J Obes 2005; 29: 1452-1456.

3. Williams IL, Wheatcroft SB, Shah AM, Kearney MT. Obesity, atherosclerosis and the vascular endothelium: mechanisms of reduced nitric oxide bioavailability in obese humans. Int $J$ Obes Relat Metab Disord 2002; 26: 754-764.

4. Nathan C. Inducible nitric oxide synthase: what difference does it make? J Clin Invest 1997; 100: 2417-2423.

5. Gunnett CA, Heistad DD, Faraci FM. Gene-targeted mice reveal a critical role for inducible nitric oxide synthase in vascular dysfunction during diabetes. Stroke 2003; 34: 2970-2974.

6. Zahedi AsI S, Ghasemi A, Azizi F. Serum nitric oxide metabolites in subjects with metabolic syndrome. Clin Biochem 2008; 41: 1342-1347.

7. Executive Summary of the Third Report of the National Cholesterol Education Program (NCEP) Expert Panel on Detection, Evaluation, and Treatment of High Blood Cholesterol in Adults (Adult Treatment Panel III). JAMA 2001; 285: 2486-2497.

8. Haffner SM. Insulin resistance, inflammation, and the prediabetic state. Am J Cardiol 2003; 92: 18J-26J.

9. Guevara I, Iwanejko J, Dembinska-Kiec A, Pankiewicz J, levels in patients with severe obesity (mean BMI $=39 \mathrm{~kg} /$ $\mathrm{m}^{2}$ ). However, in that study $\mathrm{NO}$ and adiponectin levels were correlated in normal weight subjects (mean BMI $=21.1 \mathrm{~kg}$ / $\mathrm{m}^{2}$ ), and the authors concluded that in non-obese subjects adiponectin could enhance eNOS activity and contribute to endogenous NO generation (15). On the other hand, our results did not show a correlation between adiponectin and $\mathrm{NOx}$ in control subjects $\left(\mathrm{BMI}=23.8 \pm 3.1 \mathrm{~kg} / \mathrm{m}^{2}\right)$, perhaps because of our small sample.

When considering the results of the present study, the relatively small number of subjects (50 in each group), because only few individuals met the rigorous criteria for inclusion in these groups, and wide dispersion of data are limitations that have to be seriously considered.

Nevertheless, the data suggest that there seems to be a close correlation between serum NO levels and changes in the characteristic parameters related to obesity, such as insulin resistance, oxidative stress and inflammatory markers. Then, it is conceivable to assume that in MS patients a redox imbalance, characterized by increased plasma oxidation and reduced antioxidant capacity, may contribute to a worsening of the clinical picture.

\section{Acknowledgments}

Research supported by CNPq.

Wanat A, Anna P, et al. Determination of nitrite/nitrate in human biological material by the simple Griess reaction. Clin Chim Acta 1998; 274: 177-188.

10. Navarro-Gonzalvez JA, Garcia-Benayas C, Arenas J. Semiautomated measurement of nitrate in biological fluids. Clin Chem 1998; 44: 679-681.

11. Gonzalez FB, Llesuy S, Boveris A. Hydroperoxide-initiated chemiluminescence: an assay for oxidative stress in biopsies of heart, liver, and muscle. Free Radic Biol Med 1991; 10: $93-100$

12. Casado MF, Cecchini AL, Simao AN, Oliveira RD, Cecchini $\mathrm{R}$. Free radical-mediated pre-hemolytic injury in human red blood cells subjected to lead acetate as evaluated by chemiluminescence. Food Chem Toxicol 2007; 45: 945-952.

13. Cecchini R, Aruoma OI, Halliwell B. The action of hydrogen peroxide on the formation of thiobarbituric acid-reactive material from microsomes, liposomes or from DNA damaged by bleomycin or phenanthroline. Artefacts in the thiobarbituric acid test. Free Radic Res Commun 1990; 10: 245-258.

14. Sun $Y X$, Hu SJ, Zhang $X H$, Sun J, Zhu CH, Zhang ZJ. [Plasma levels of VWF and NO in patients with metabolic syndrome and their relationship with metabolic disorders]. Zhejiang Da Xue Xue Bao Yi Xue Ban 2006; 35: 315-318.

15. Lin LY, Lee WJ, Shen HN, Yang WS, Pai NH, Su TC, et al. Nitric oxide production is paradoxically decreased after weight reduction surgery in morbid obesity patients. Atherosclerosis 2007; 190: 436-442. 
16. Ghasemi A, ZahediasI S, Azizi F. Reference values for serum nitric oxide metabolites in an adult population. Clin Biochem 2010; 43: 89-94.

17. Urakawa H, Katsuki A, Sumida Y, Gabazza EC, Murashima $\mathrm{S}$, Morioka $\mathrm{K}$, et al. Oxidative stress is associated with adiposity and insulin resistance in men. $J$ Clin Endocrinol Metab 2003; 88: 4673-4676.

18. Lee KU. Oxidative stress markers in Korean subjects with insulin resistance syndrome. Diabetes Res Clin Pract 2001; 54 (Suppl 2): S29-S33.

19. Skalicky J, Muzakova V, Kandar R, Meloun M, Rousar T, Palicka V. Evaluation of oxidative stress and inflammation in obese adults with metabolic syndrome. Clin Chem Lab Med 2008; 46: 499-505.

20. Gersch C, Palii SP, Kim KM, Angerhofer A, Johnson RJ, Henderson GN. Inactivation of nitric oxide by uric acid. Nucleosides Nucleotides Nucleic Acids 2008; 27: 967-978.

21. Tao L, Gao E, Jiao X, Yuan Y, Li S, Christopher TA, et al. Adiponectin cardioprotection after myocardial ischemia/reperfusion involves the reduction of oxidative/nitrative stress. Circulation 2007; 115: 1408-1416.

22. Li R, Wang WQ, Zhang H, Yang X, Fan Q, Christopher TA, et al. Adiponectin improves endothelial function in hyperlipidemic rats by reducing oxidative/nitrative stress and differential regulation of eNOS/iNOS activity. Am J Physiol Endocrinol Metab 2007; 293: E1703-E1708.

23. Yamaguchi Y, Yoshikawa N, Kagota S, Nakamura K, Haginaka J, Kunitomo M. Elevated circulating levels of markers of oxidative-nitrative stress and inflammation in a genetic rat model of metabolic syndrome. Nitric Oxide 2006; 15: 380386.

24. Esposito K, Ciotola M, Schisano B, Misso L, Giannetti G, Ceriello A, et al. Oxidative stress in the metabolic syndrome. $J$ Endocrinol Invest 2006; 29: 791-795.

25. Nakagawa T, Hu H, Zharikov S, Tuttle KR, Short RA, Glushakova $\mathrm{O}$, et al. A causal role for uric acid in fructose-induced metabolic syndrome. Am J Physiol Renal Physiol 2006; 290: F625-F631.

26. Imaram W, Gersch C, Kim KM, Johnson RJ, Henderson GN, Angerhofer A. Radicals in the reaction between peroxynitrite and uric acid identified by electron spin resonance spectroscopy and liquid chromatography mass spectrometry. Free Radic Biol Med 2010; 49: 275-281.

27. Johnson RJ, Rivard C, Nakagawa T, Sautin YY, SanchezLozada LG. Uric acid: more to learn, more experiments to do. Am J Hypertens 2009; 22: 952-953.

28. Zharikov S, Krotova K, Hu H, Baylis C, Johnson RJ, Block $E R$, et al. Uric acid decreases NO production and increases arginase activity in cultured pulmonary artery endothelial cells. Am J Physiol Cell Physiol 2008; 295: C1183-C1190.

29. Simao AN, Dichi JB, Barbosa DS, Cecchini R, Dichi I. Influence of uric acid and gamma-glutamyltransferase on total antioxidant capacity and oxidative stress in patients with metabolic syndrome. Nutrition 2008; 24: 675-681.

30. Fried SK, Bunkin DA, Greenberg AS. Omental and subcutaneous adipose tissues of obese subjects release interleukin-6: depot difference and regulation by glucocorticoid. $J$ Clin Endocrinol Metab 1998; 83: 847-850.

31. Mohamed-Ali V, Goodrick S, Rawesh A, Katz DR, Miles JM, Yudkin JS, et al. Subcutaneous adipose tissue releases interleukin-6, but not tumor necrosis factor-alpha, in vivo. $J$ Clin Endocrinol Metab 1997; 82: 4196-4200.

32. Kern PA, Ranganathan S, Li C, Wood L, Ranganathan $\mathrm{G}$. Adipose tissue tumor necrosis factor and interleukin-6 expression in human obesity and insulin resistance. Am J Physiol Endocrinol Metab 2001; 280: E745-E751.

33. Matsushita K, Yatsuya H, Tamakoshi K, Wada K, Otsuka R, Takefuji S, et al. Comparison of circulating adiponectin and proinflammatory markers regarding their association with metabolic syndrome in Japanese men. Arterioscler Thromb Vasc Biol 2006; 26: 871-876. 